\section{Revue de Sémantique et Pragmatique}

35-36 | 2015

Interface sémantique/morphologie

\title{
Morphologie, sémantique, syntaxe, discours du statut ambigu des « noms » désadjectivaux en [le adjectif]
}

Morphology, semantics, syntax, discourse. Ambiguous status of desadjectival « nouns » in (fr.) [le Adjectif]

Michelle Lecolle

\section{OpenEdition \\ Journals}

Édition électronique

URL : http://journals.openedition.org/rsp/1416

DOI : $10.4000 /$ rsp. 1416

ISSN : 2610-4377

Éditeur

Presses universitaires d'Orléans

Édition imprimée

Date de publication : 1 mars 2015

Pagination : $27-47$

ISSN : 1285-4093

Référence électronique

Michelle Lecolle, "Morphologie, sémantique, syntaxe, discours du statut ambigu des «noms »

désadjectivaux en [le adjectif] », Revue de Sémantique et Pragmatique [En ligne], 35-36 | 2015, mis en ligne le 01 mars 2016, consulté le 09 mai 2020. URL : http://journals.openedition.org/rsp/1416 ; DOI : https://doi.org/10.4000/rsp.1416 


\section{MORPHOLOGIE, SÉMANTIQUE, SYNTAXE, DISCOURS DU STATUT AMBIGU DES « NOMS » DÉSADJECTIVAUX EN [LE ADJECTIF]}

Michelle Lecolle

Université de Lorraine, CREM-Praxitexte

Cet article se propose d'étudier et de discuter le statut théorique et les réalisations de la structure en [le $\mathrm{Adj}]$, dont voici quelques exemples :

(1) Le social et le politique se tiennent et se soutiennent (L'Express, 10-16 mars 1969)

(2) Rien ne nous permet de comprendre les relations de la conscience et des processus nerveux, du mental et du cérébral (Carrel, L'Homme, 1935).

(3) L'État est d'abord une médiation entre l'individuel et le social, entre le privé (les intérêts privés) et le public (l'intérêt général) (Traité sociol., 1968).

(4) L'industrie des médias à l'ère du numérique (titre de colloque)

(5) Le cru et le cuit (ouvrage de Levi Strauss)

où l'on peut gloser [le Adj] par 'ce qui est Adj' - 'ce qui est individuel/public/ social/politique/mental'... Les réalisations de ce type sont nommées « emplois substantivés d'adjectif » ou « adjectifs substantivés à valeur de neutre » dans le Trésor de la langue Française (TLF ${ }^{1}$ ), ce qui oriente vers un phénomène discursif. Mais on peut aussi en parler en termes de "nominalisation désadjectivale », c'est-à-dire de construction morphologique, dans la mesure où le procédé est comparable à d'autres nominalisations, dérivées - blanc $>$ la blancheur vs le blanc -, ou non - politique > la politique vs le politique. Le terme de "nominalisation désadjectivale par conversion » (dérivation

${ }^{1}$ Consulté à partir du TLFi : ATILF, CNRS. Le Trésor de la Langue Française Informatisé (TLFi) . [en ligne] http://atilf.atilf.fr/tlf.htm 
non affixale chez Kerleroux 1996 et 1999) peut aussi être avancé selon un autre point de vue, si on envisage, comme nous le faisons, la construction de certains lexèmes - (un, l') humanitaire, (un, le) politique, (un, le) nécessaire, (un, le) matériel - comme l'issue d'un processus de substantivation au départ conjoncturel et syntaxiquement régulier - « syntactico-discursif ». L'adoption du terme de nominalisation par conversion comme celle, plus générale, de «transcatégorisation » (cf. Lecolle 2012) signifierait la prise en compte de ces structures dans le cadre de la description morphologique, et c'est bien cette question qui est mise ici en discussion. De fait, ces réalisations ont un statut intermédiaire : d'une part, la structure elle-même peut, selon les cas, se situer entre des faits discursifs purement conjoncturels - avec l'inconnu, l'extraordinaire, le cru, l'individuel - et des faits de lexique (construction de lexème); d'autre part, l'ambiguïté catégorielle (entre Adj et $\mathrm{N}$ ) des lexèmes qui y entrent témoigne de la facilité du passage entre les catégories Adjectif et Nom et, pour tout dire, de la porosité de la frontière de l'une à l'autre (cf. Roché 2003, Noailly 2004). C'est donc en tant qu'objet hybride que nous abordons cette question, qui, selon nous, engage une démarche articulant plusieurs champs de la linguistique : morphologie, sémantique lexicale, mais aussi syntaxe et discours.

À l'observation, la structure en [le Adj] s'avère assez prolifique ${ }^{2}$; on la trouve dans le discours philosophique et scientifique, mais aussi dans la presse, dans des titres de colloques ou d'ouvrages en sciences sociales, dans les textes littéraires. En reprenant le terme qu'utilise Sablayrolles (2000) à propos des néologismes, on peut parler de cette structure comme d'une «matrice » ( " matrice lexicogénique » chez l'auteur) ou, dans nos termes, comme d'un « moule » constructionnel : parler de moule, de matrice ou encore de construction, comme Lauwers (2008), dans la perspective de la Grammaire des Constructions, permet de souligner son caractère régulier d'une part, accueillant et productif d'autre part.

Dans une première partie, nous délimiterons et caractériserons l'objet d'étude - l'Adj substantivé et ses différentes potentialités de réalisations -, à travers des exemples issus de notre collecte. Après la présentation de celleci, une deuxième partie sera consacrée à une proposition de répartition des réalisations observées, dans laquelle entrent comme deux extrêmes des réalisations discursives conjoncturelles d'un côté (e.g. l'extraordinaire), et des créations avérées de lexème par nominalisation (e.g. un, l'humanitaire ; un, le public) de l'autre. In fine, il s'agit d'examiner la polycatégorialité des

2 Nous avons collecté jusqu'à présent 894 Adj différents dans cette structure, cf. présentation en $\S 2.1$. 
instanciations de la «matrice » en [le Adj] et d'en prendre en compte les enjeux, notamment sémantiques : si deux lexèmes (Adj et $\mathrm{N}$ ) cohabitent, leurs significations conservent des liens, entrainant parfois une polysémie (le public vs le privé et le public (empirique)). Et certaines de ces réalisations restent en quelque sorte « suspendues » entre les deux catégories, dans une ambigüité catégorielle fondamentale.

\section{CADRE DE DESCRIPTION DE [LE ADJ]}

Dans cette partie, nous détaillons les propriétés de l'objet, d'un point de vue sémantique et syntaxique.

\subsection{DÉLIMITATION DE L'OBJET}

Nous nommons Adjectif Substantivé (AS) le phénomène à l'étude ; cette formulation nous permet de maintenir en présence les deux catégories concernées (Adj et Subst) sans préjuger du statut de la réalisation (lexème, construction, fait de discours), qui est l'objet même de l'étude. Les AS sont un phénomène connu, qui a déjà été abordé dans différents écrits, spécifiquement comme chez Bécherel (1979), ou dans un cadre qui les englobe : des articles portant sur l'histoire des catégories grammaticales (voir Colombat 1992 (dir.)) signalent l'existence de ces réalisations dès le latin (cf. le neutre album). Kerleroux (1996), les confrontant à des conversions désadjectivales avérées, en parle en termes de "distorsion catégorielle » (voir infra). Des approches sémantiques rapportent les AS à une catégorie comme le massif (voir Riegel 2013, Lauwers 2008), ou les comparent aux noms de qualité (NQ) (Kerleroux op. cit., Lauwers op. cit., Sanchez et Cadiot 2007). Mais, signe qu'il s'agit d'un objet hybride, les AS font aussi l'objet d'approches « entre deux » : syntaxe et sémantique chez Noailly (1999 et 2004), morphologie et sémantique (Lecolle 2011), morphologie, syntaxe et sémantique (Lauwers 2008 et à paraître, Schwarze 2012), morphologie, sémantique et discours (Lecolle 2012 et à paraître) voir aussi les approches de lexicologie et morphologie de Roché (notamment 1991). Lauwers (2008 et à paraître), comme nous ici, interroge le statut et le niveau de description adéquat de ces réalisations. Abordant également le niveau lexical et la lexicalisation, il postule, pour les Adj qualificatifs, une continuité entre réalisation discursive conjoncturelle et substantivation. Si nous abordons malgré tout la question, c'est qu'elle ne nous semble pas totalement épuisée ; en nous appuyant sur les descriptions susmentionnées (et en nous en démarquant parfois), nous souhaitons pour notre part poursuivre les perspectives «inter- » ou « trans- », en faisant intervenir les différents niveaux présentés en introduction, et ceci à partir d'un inventaire conséquent de réalisations discursives et lexicales comprenant tous types d'Adj. Il ne s'agit pas non plus de clore la réflexion, et nous souhaitons au contraire ouvrir des pistes : comme il apparaîtra, de 
nombreux niveaux d'analyse s'entrecroisent, dont chacun mériterait d'être approfondi.

\subsubsection{Cadre de la morphologie constructionnelle : conversion}

Pour délimiter le phénomène, nous le situons dans un premier temps dans le cadre de la conversion ( $\mathrm{Adj}>\mathrm{N}$ ). Dans ce cadre, nous excluons de l'analyse :

- les noms de disciplines au féminin formés sur des adjectifs homophones: la politique ; la sémantique; la mathématique-les mathématiques ;

- les convers Subst issus d'Adj se rapportant aux humains et désignant un type humain (noté $[+\mathrm{H}])$ : le, un timide; le, un bavard; le, un volontaire (cf. Noailly 1999, Schwarze op. cit., Lauwers 2008, Lecolle 2011 et 2012), qui relèvent d'un autre type de conversion désadjectivale. Ce qui n'exclut pas la possibilité de gentil, sémillant, solennel dans :

(6) Il diffère de son rival à chaque pas, de toute la distance du gentil et du sémillant au solennel (Sainte-Beuve, Port-Royal).

De cet exemple et de quelques autres (avec libertin, prudent, dégingandé...), nous concluons que le trait $[+\mathrm{H}]$ de l'Adj ne s'oppose pas à ces réalisations, pourvu qu'elles puissent construire une catégorie (celle du « gentil »- 'ce qui est gentil', etc. -, voir les descriptions sémantiques infra).

Toujours dans un cadre de morphologie constructionnelle, nous comparerons nos structures en [le Adj] et les « Subst» output à des N tels que (le) calme, (le) vide, (le) trouble, (le) sérieux, auxquels Kerleroux (op. cit.) réserve le statut de Subst désadjectivaux ; pour ceux-ci, la conversion désadjectivale est équivalente à la dérivation affixale qu'on trouve avec les NQ. Il s'agit d'ailleurs pour Kerleroux d'un même phénomène de dérivation, mais sans affixe ${ }^{3}$ :

beau $_{\text {Adj }}>$ beauté $_{\text {subst }} ;$ triste $_{\text {Adj }}>$ tristesse $_{\text {subst }} ;$ calme $_{\text {Adj }}>$ calme subst $_{1}$

Contrairement aux NQ calme, trouble, vide, sérieux (et beauté, tristesse), les AS n'ont pas toutes les propriétés nominales. Ils correspondent à ce que Kerleroux nomme une « distorsion catégorielle », c'est-à-dire qu'ils occupent syntaxiquement une position de noms, alors qu' " [ils] n'acquièrent pas les propriétés d'identité catégorielle, pleine et nouvelle, que procure le statut de dérivé morphologique » (p. 189). L'auteure propose de nombreux tests à l'appui de cette démonstration. Un autre test a pu être proposé pour illustrer la transposition d'Adj à NQ dérivé, avec conservation du sens (Riegel 2004, Koehl 2012). À partir de calme, sérieux, puis beau, public, qui ont également un Subst dérivé :

Son comportement est calme/sérieux / son visage est beau / le débat est public

Le calme/sérieux de son comportement / la beauté de son visage / la publicité du débat

\footnotetext{
${ }^{3}$ Cette répartition est généralement adoptée (cf. notamment Roché 2005, Koehl 2012).
} 
L'existence de ces NQ dérivés permet qu'on les compare aux AS. Par exemple :

La beauté de son visage / *le beau de son visage

La publicité du débat $=$ Le caractère public du débat $/ *$ Le public du débat.

Ces descriptions sont opératoires et nous serviront de repère. Mais les champs ne sont pas toujours aussi nettement départagés, que ce soit syntaxiquement (voir $§ 1.4$.) ou sémantiquement. La question de la répartition entre AS et NQ nécessiterait des études approfondies qui, à notre connaissance, n'ont été faites que sur quelques mots (beau/beauté, blanc/blancheur par exemple). Nous livrerons plus bas quelques éléments concernant cette question (cf. § 2.2.2).

\subsection{2. [le Adj] dans le cadre syntactico-discursif}

La section précédente confrontait [le Adj] à la construction de lexème désadjectival (conversion ou dérivation). Cette section interroge la structure elle-même. On pourrait être tenté d'interpréter la signification des AS comme résultant de l'élision d'un nom-tête : que ce soit dans des emplois en discours, ou pour des lexèmes, après lexicalisation, cette solution a été avancée. Si elle convient pour les adjectifs nominalisés désignant des types humains tels que présentés ci-dessus (un (homme) bavard) ou dans d'autres cas de dénomination univoque d'un individu (le (champignon) laminaire, exemple de Lecolle 2011), cette solution ne tient pas dans le cas présent, comme l'a bien montré Noailly (1999) : en effet, quel nom tête reconstituer, si ce n'est un nom tellement général qu'il serait équivalent à 'ce qui est' (+ Adj) ? On peut aussi, pour s'en convaincre, comparer des AS à des exemples d'ellipses discursives effectives, c'est-à-dire des cas d'anaphores où le nom tête est élidé, parce que présent dans le contexte :

(7) Batteue distingue deux ordres de construction [de phrase] : le métaphysique, qui n'est autre que le traditionnel ordre sujet-prédicat, et le moral, « fondé sur l'intérêt de la personne qui parle». (Genette, Mimologiques, 1976, p. 227)

(8) Le bon sens, c'est de sortir des énergies fossiles pour aller vers les renouvelables. (Laurence Rossignol, sénatrice, Politis 6/12/2012)

L'interprétation est différente : ici, non pas 'ce qui est métaphysique, moral' (AS), mais 'l'ordre de construction métaphysique' et 'l'ordre de construction moral' ${ }^{4}$.

Au niveau du lexème et de la lexicalisation, il s'agit là aussi d'un cas différent de celui de l'AS : à titre d'exemple, on comparera deux réalisations de [le Adj] relevant du même domaine : l'exécutif, équivalent à 'le gouvernement' peut être interprété comme une ellipse parce que lexicalisé d'une part, circonscrit

\footnotetext{
${ }^{4}$ Voir des remarques similaires chez Lauwers (2008 : $138-139$ et 142).
} 
et univoque d'autre part - « le pouvoir exécutif ». En regard, le législatif n'a pas d'entrée nominale dans les dictionnaires ${ }^{5}$, et reste (pour le moment) une réalisation adjectivale en AS.

\subsection{PRÉSENTATION DE LA STRUCTURE « MATRICIELLE » [LE ADJ]}

L'adjectif est généralement décrit comme sémantiquement dépendant (voir notamment Roché 2004, Riegel 2004, Schwarze 2012) ; c'est, dans les termes de ce dernier, « un prédicat qui en requiert un autre [le substantif]» (p. 163). Lors d'un passage de Adj à Subst, il y a donc passage à l'indépendance, et c'est bien ce qui arrive dans les cas de conversion, par exemple entre timide $_{A d j}$ et timide $_{\text {subst }}$ ou entre calme $e_{\text {Adj }}$ et calme $e_{\text {subss }}$ " conçus comme des 'objets de pensée' que l'on peut évoquer en tant que tels » (Riegel 2004).

Venons-en aux AS. Les concernant, l'indépendance que mentionne Schwarze n'est pas totalement avérée, d'un point de vue syntaxique : ni changement de déterminant (uniquement $l e$ et $d u$, voir infra), ni pluralisation ne sont possibles. Pas d'indépendance non plus du point de vue sémantique : [le Adj] peut être glosé par 'ce qui est Adj' (le social : 'ce qui est social'); son sens reste donc celui de l'Adj. Mais le passage à un emploi nominal lui permet dans le même temps d'être objet de référence, d'un certain type. Les descriptions sémantiques tournent autour de la même idée chez différents auteurs : le passage Adj/[le Adj] correspond à une " massification », à une « homogénéisation» (Lauwers 2008); et le sens de [le Adj] ('ce qui est Adj') renvoie à du continu - indéfini, massif, collectif (potentiellement, 'l'ensemble de ce qui est Adj') (Roché 1991, Noailly 1999 et 2004, Lecolle 2011 et 2012, Riegel 2013). Selon Noailly (1999 : 140), « Le Adj fonctionne comme une sorte de valeur générique, et vise toute référence potentielle répondant à une propriété ». De là, s'ouvre une diversité de réalisations possibles, selon la signification des lexèmes adjectivaux et les contextes. Ainsi, comme le signale également Lauwers (2008), on peut expliquer à partir de [le Adj] deux types de réalisations :

- $[d u \mathrm{Adj}]$ correspond à un découpage, une partition sur la référence continue construite par le Adj - ce que nous avons nommé un « prélèvement » (Lecolle 2011) : du neuf/du lourd/du commun réfère à une partie de la masse que dénomme le neuflle lourd/le commun - (9) et (10) :

(9) Aujourd'hui une première émission estivale avec du neuf, du vieux, du lourd, du léger, du freestyle [...] (radio campus, exemple de Goes 2011)

(10) $[\ldots]$ ceux qui aspirent à reconstituer du commun (France Culture, 12/2013)

\footnotetext{
${ }^{5}$ Petit Robert (2012), Dictionnaire de l'Académie 9e édition, TLFi.
} 
- Les lexèmes nominaux, e.g. un politique, un scolaire, un blanc, un imprévu (individu) relèvent aussi de ce que nous nommons un « prélèvement », sous la forme ici d'une « singularisation », d'une individuation à partir de la propriété (massive) constituée par l'Adj substantivé.

\subsection{PROPRIÉTÉS MORPHOSYNTAXIQUES DES ADJECTIFS SUBSTANTIVÉS}

Des réalisations comparables aux AS ont été mentionnées pour l'allemand (Schwarze op. cit.) et décrites en espagnol (Villalba 2009). Dans les deux cas, on peut souligner la présence d'un genre «neutre », qui se distingue, donc, du masculin.

Pour l'espagnol, une structure telle que [le Adj] se réalise en [lo + Adj] $(l o \neq \mathrm{el})$

(11) lo definito y seguro est traduit par "definitive and sure things" ("ce qui est sûr et définitif')

D'après les exemples de Villalba, il semble que [lo + Adj] ne recouvre pas le même champ qu'en français : des structures impossibles en français paraissent être possibles en espagnol, et l'AS espagnol recoupe le NQ affixé (e.g. la estrechez vs lo estrecho), même si, en définitive, il n'en connait pas toutes les potentialités.

En allemand (cf. Schwarze op. cit.), on peut opposer das Klassische ('le classique') à der modernste Klassiker ('le classique le plus moderne', comme type d'individu) :

(12) Le classique se vend bien. - $\underline{\text { Das }}$ Klassische verkauft sich gut.

Dans ces deux cas, c'est le neutre qui se réalise, par le biais d'un marquage différent du masculin. Le français n'a pas de genre neutre marqué, mais on parle parfois de «pseudo-neutre » (Roché 1991). De fait, on sent bien la différence de le dans [le Adj] avec le masculin, du point de vue du sens - cf. le rapprochement avec le massif. C'est aussi, on peut le supposer, le sens de la glose du TLF « adjectif substantivé à valeur de neutre », reprise de la tradition.

Pour ce qui est du nombre, les AS possèdent une unicité intrinsèque.

\subsection{STRUCTURES SYNTAXIQUES}

Nous détaillons dans cette partie des exemples d'AS, en nous intéressant à la structure interne des syntagmes ${ }^{6}$, aux fonctions syntaxiques qu'ils occupent, à la signification des Adj et à l'incidence de ces différents niveaux d'analyse sur le sens.

\footnotetext{
${ }^{6}$ Voir aussi sur ce point les analyses de Lauwers (2008) et de Schwarze (op. cit. : 155-156).
} 
1. Reprenons tout d'abord la structure [le Adj de dét N]. Elle a été mentionnée en 1.1.1 comme illustrant le cas des NQ dérivés avec ou sans affixe (cf. le calme de son comportement, la beauté de son visage), qu'elle est censée délimiter. Or, les exemples (13)-(15) attestent de la possibilité de cette même structure avec des AS, mais le sens qui en découle est différent. Observons ceci : en (13)-(14), on interprète le tendre/ le maigre/ le fort/ le faible de non pas comme 'la tendreté/ maigreur/ force/ faiblesse de', mais comme : 'ce qui est tendre/ maigre/ blanc/ fort/ faible dans', c'est-à-dire : 'la partie tendre/ maigre/ blanche/ forte/ faible de'. Ici, les Adj en AS délimitent des parties ou des catégories dans un tout.

(13) le tendre des aisselles (Giono, Que ma joie demeure, 1935) / Le maigre d'un jambon / Le blanc d'un poulet

(14) enfin, après avoir examiné le fort et le faible des sciences, il fut décidé que Monsieur le marquis apprendrait à danser (Voltaire)

Un cas différent apparaît avec des Adj évaluatifs dans les énoncés (15) :

(15) l'odieux de sa conduite / le remarquable de l'affaire (cités par Bécherel) / le tragique de la situation

qui s'interprètent de deux manières différentes :

(i) 'ce qui est odieux/ remarquable/ tragique dans', comme en (13) et (14) - c'est ce qu'on nomme une «valeur d'occurrence » (Noailly 1999);

(ii) comme un NQ, qu'on glosera par 'le caractère odieux/ remarquable/ tragique de', et qui correspond à la transposition mentionnée en 1.1.1 et rappelée ci-dessus (cf. sa conduite est odieusel l'odieux de sa conduite, sur le modèle de son visage est beaul la beauté de son visage) - interprétation qu'on n'a pas en (13)-(14). On pourrait ajouter aux Adj représentés dans (15) d'autres Adj qualitatifs évaluatifs inouï, extraordinaire, singulier, difficile.

2. Ces mêmes Adj évaluatifs entrent dans les structures [le Adj c'est/ est $d e+$ Vinf] et [le Adj c'est/ est que + subordonnée] :

Le difficile/ l'extraordinaire/ le piquant/ le pittoresque/ l'inquiétant/ le singulier/ l'inédit c'est de/ est que/, c'est que [...]

Dans tous ces cas, on peut insérer un adverbe de degré dans le syntagme : le plus/ vraiment difficile/ singulier/ chic c'est del c'est que [...].

(16) Le singulier est que la question, aujourd'hui, puisse se poser (M. Bloch, 1944).

(17) Le difficile, c'est de s'arrêter (cité par Bécherel)

(18) Le chic, c'est de se relever / le chic, c'est de ne pas faire comme les autres / le chic, c'est de réussir à rester mince. (internet)

Remarquons que ces structures ne fonctionnent pas avec tous types d'Adj : ? Le politique, le social / le sale, le maigre / le public est que/ c'est de/ c'est que [...] 
Ces structures relèvent de ce que Legallois (2008) nomme des « constructions spécificationnelles » (CS); dans celles-ci entrent, selon l'étude? des $\mathrm{N}$ tels que vérité, objectif, conséquence, problème, paradoxe, souhait, dont le point commun est d'être "sous-spécifiés » (NSS, abréviation de l'auteur), mais aussi des Adj, ceux-là mêmes que nous présentons ci-dessus : important, essentiel, étonnant, inouï, extraordinaire. L'auteur intègre l'ensemble de ces données, noms et adjectifs, en parlant d'emploi nominal, ce à quoi nous ne souscrivons pas - les Adj dans cette structure restent des Adj. Mais l'essentiel ici est que la structure spécificationnelle révèle une caractéristique sémantique commune à nos Adj et aux NSS : à la différence des AS comme le politique, le social, le sale, le maigre mentionnés ci-dessus comme exclus de la structure, les Adj et les $\mathrm{N}$ présentés dans l'étude ont pour point commun de ne pas délimiter une classe notionnelle « objective » ou objectivable, mais plutôt de proposer un point de vue, propre à être éventuellement argumenté. De par leur signification, les Adj évaluatifs présentent naturellement une affinité avec le sens dégagé par une telle structure.

3. L'AS constitue un syntagme, qui occupe une fonction référentielle : sujet, COD, COI, complément de nom.

(19) L'outré, le caricatural, n'[...] épargnent aucun personnage (Colette)

(20) Quelle différence mettez-vous entre le romanesque et l'exagéré ? (cité par Bécherel)

(21) Par quelles phases s'accomplit la liaison du moral et du religieux, dans les sociétés anciennes? (Traité sociol., 1968).

Dans ces fonctions, [le Adj] s'interprète comme 'la classe de ce qui est Adj', dont les contours sont délimités par le sens de l'Adj. L'extension de l'AS peut alors correspondre à une notion, une catégorie de pensée, de classement - celle du « moral », du « religieux », du « romanesque » -, mais, avec des adjectifs comme inconnu, outré, détestable, excellent, grotesque, horrible, il s'interprète comme une classe conjoncturelle et appréciative, celle mise en place discursivement par l'énonciateur. Nous avons rencontré précédemment avec les NSS (cf. 2 supra), des exemples similaires, mais il nous semble par comparaison que, dans le cas présent, les fonctions sujet, COD, COI occupées par les $\mathrm{SN}$ en [le Adj] « tirent» l'interprétation de ces classes appréciatives vers une objectivation du référent - objectivation qui n'est, malgré tout, pas atteinte, du moins avec les Adj appréciatifs ${ }^{8}$.

En résumé, on confirme avec ces séries de structures que les AS sont, par essence, dans l'« entre-deux », une zone floue où la signification de l'Adj

\footnotetext{
${ }^{7}$ En corpus de presse, 214 et 208 «noms », dans les structures c'est de + Inf et c'est que.

${ }^{8}$ Sauf cas de lexicalisation (voir infra $§ 2.2$ « série $2 »$ ).
} 
(catégorisant ou appréciatif dans nos exemples), la structure d'AS, la fonction syntaxique et le cotexte phrastique voire textuel interagissent. On peut aussi remarquer et discuter la fluctuation de l'interprétation pour un même Adj, et la difficulté à constituer des ensembles sémantiques homogènes d'Adj. Tous ces obstacles tiennent, selon nous, à l'objet même : les AS ne se contentent pas de mettre en œuvre des Adj; ils profilent, voire remanient leur signification.

\section{RÉALISATIONS D'ADJECTIFS SUBSTANTIVÉS}

Nous exposons à présent notre inventaire d'AS (§ 2.1), avant d'en proposer une catégorisation $(\$ 2.2)$. Sur la base d'exemples discursifs et de lexèmes attestés, nous discutons ensuite la continuité et les interférences entre ces catégories $(\S 2.3)$.

\subsection{RESSOURCES ET INVENTAIRE}

Pour étendre notre analyse à la plus grande diversité possible, nous avons procédé à une collecte de [le Adj]. Une partie provient d'énoncés rencontrés au quotidien - comme signalé en introduction, les textes de sciences humaines, d'un côté, la presse de l'autre sont de bons pourvoyeurs. Une autre partie, la plus importante, a été recueillie à partir du TLFi en croisant les critères de recherche automatiques suivants : a) Adj dans «catégorie grammaticale »; b) dans « indicateur » présence du mot neutre, qui apparaît dans les expressions définitoires signalées en introduction - « emploi substantivé masculin à valeur de neutre ». Le résultat est de 1080 exemples, correspondant à 830 Adj différents (plusieurs exemples par Adj). Nous y avons ajouté les exemples provenant de diverses publications (Bécherel 1979, Noailly 2004, Riegel 2013). Le nombre total d'items est, pour le moment, de 894, mais la liste reste ouverte. Ces résultats sont à envisager comme une source d'attestations et non réellement comme un corpus, étant donné le caractère plutôt monolithique, daté et stylistiquement marqué des exemples du TLF ( $v s$ nos relevés contemporains) : on peut se demander ce qu'ils reflètent de la pratique contemporaine réelle ${ }^{9}$; étant donné aussi que nous n'avons pas (encore) exploré davantage la ressource de ce dictionnaire. Les « résultats » qui suivent résultent d'observations d'ensemble et de quelques décomptes.

Une constatation générale : étant donné le nombre et la diversité de ces Adj, il parait difficile de dire ce qui ne peut pas entrer dans les AS - sans doute

${ }^{9}$ Les exemples du TLF consultés sont plutôt issus de discours normés (littérature, essais). On sait que le projet du dictionnaire faisait porter la nomenclature sur une période débutant à la fin du XIX ${ }^{\mathrm{e}}$ siècle. De fait, les exemples consultés sont plutôt anciens au regard de la période concernée. Pas non plus d'exemples réellement contemporains ( $\mathrm{XXI}^{\mathrm{e}}$ siècle), puisque le TLF n'est pas renouvelé. 
les Adj $[+\mathrm{H}]$ cités plus haut, mais ce serait à confirmer. De fait, en remaniant le sens des Adj, la structure [le Adj] en remanie aussi les potentialités. À cela, il faut ajouter le rôle du cotexte antérieur (supposé) et celui de certaines structures (énumérations - e.g. (6), (9) -, constructions binaires - e.g. (20), (21) $)^{10}$ : ainsi favorisés, des $\operatorname{Adj}[+\mathrm{H}]$ apparaissent là où ils n'étaient pas attendus (cf. § 1.1.1).

Voici quelques types d'adjectifs représentés :

- Adj qualificatifs « objectifs » (et notamment des Adj de couleur) :

(22) l'humide et le sec sont les deux ennemis du vigneron (France, Rôtisserie, 1893)

- Adj qualificatifs évaluatifs (extraordinaire, banal, difficile, stupide, fade, lugubre) ;

- participes passés (12,3\% : jugé, lâché, lustré, stylisé, tourmenté, fini, exagéré) :

(23) Le contourné, le chargé d'ornements nous paraît sur le champ trop petit. (Stendhal)

- participes présents $(4,8 \%$ : larmoyant, menaçant, existant, étouffant, saignant) :

(24) Autrefois j'ai identifié l'existant avec le repérable (Marcel, Journal, 1923).

- les Adj en -ablel-ible sur base verbale (nommable, saisissable, tolérable, pensable, traduisible) sont en nombre relativement important : 14,4\%. Parmi ces dérivés, 70 préfixés en in- («in-Xable » : incomparable, incompressible, incroyable : $\left.7,8 \%{ }^{11}\right)$ :

(25) Renverser l'insoutenable (titre d'un ouvrage d'Yves Citton, 2012)

- La présence non négligeable d'Adj relationnels est à souligner (10\%) - ce qui contredit une remarque de Noailly (1999 : 140) - moléculaire, journalier, scientifique, scolaire, limoneux, légal, humain, alimentaire, charnel, humanitaire, urbain. Parmi eux, des Adj relationnels catégorisants (scientifique, historique, social) :

(26) Zola se trompe dans le scientifique comme Flaubert dans l'historique, parce que tous deux vont chercher trop loin (Arts et litt., 1935).

${ }^{10} \mathrm{Il}$ faudrait étudier plus précisément les structures des énoncés, et, notamment, leurs caractéristiques rhétoriques. Une étude complémentaire pourrait également aborder la construction dans un espace textuel de la référence «nominale » de certains AS à partir d'Adj : par exemple de l'Adj jouable à le jouable (attesté - voir Lecolle à paraitre). Dans cet article, nous en restons essentiellement aux AS et Adj, et à un empan phrastique.

11 Nous mentionnons ces chiffres parce que ces dérivés nous paraissent avoir un sens... remarquable, et, du moins pour les préfixés en in-, des potentialités superlatives (voir Apothéloz 2003) qui présentent des affinités avec certains attendus sémantiques des AS. L'étude devrait bien sûr être complétée, et étendue à d'autres corpus : ainsi, simple conjoncture, des Adj pourtant prévisibles comme inoubliable ne figurent pas dans les données TLFi. 
L'étiquette «Adj relationnel » est à prendre avec distance : certains ne restent pas « relationnels », et c'est parfois précisément leur emploi en AS qui favorise ce glissement.

(27) Oh! comme l'âme est faite pour la monotonie, le journalier, l'habitude! Tout ce mystère enchante, déçoit, ravit, excède tour à tour (Tharaud, Fête arabe, 1912).

Certains types d'Adj présentés précédemment peuvent être mis en rapport avec les « catégories » que nous présentons à présent.

\subsection{RÉALISATIONS D'ADJECTIFS SUBSTANTIVÉS : DU SYNTACTICO-DISCURSIF AU LEXICAL}

Nous exposons d'abord les réalisations possibles - de la réalisation conjoncturelle au lexème nominal -, puis nous discutons ces catégories, à partir d'exemples attestés.

\subsubsection{Types de réalisations possibles}

La catégorisation proposée dans le tableau 1 ci-dessous porte, non plus sur les Adj mais sur les AS. Elle cherche à contraster les types de réalisations qui découlent de cette structure : phénomène discursif, lexicalisation et types de lexicalisation. La première ligne correspond aux cas qui restent au stade de l'AS (inoü̈, flou). Les autres lignes mentionnent des réalisations en noms de domaines ou de catégories et/ou en $\mathrm{N}$ d'individus (lignes 4 et 5) ou de collectifs (ligne 6). La présence des items dans deux colonnes ou plus montre que l'Adj employé en AS coexiste avec le nom de domaine et/ou le $\mathrm{N}$ d'individu ou collectif : selon l'approche de la morphologie lexématique, ils sont alors homonymes (Kerleroux 1999).

\begin{tabular}{|c|c|c|c|c|c|}
\hline & 1. Adj & 2. AS & 3. Ndomaine, Ncatégorie & 4. Nindividu & 5. Ncoll \\
\hline 1 & inouï, flou & $\begin{array}{l}\text { l'inouï, le } \\
\text { flou }\end{array}$ & / & / & / \\
\hline 2 & numérique & le numérique & le numérique & l & I \\
\hline 3 & $\begin{array}{l}\text { uniforme } \\
\text { imprévu }\end{array}$ & $\begin{array}{l}\text { l'uniforme } \\
\text { l'imprévu }\end{array}$ & 1 & $\begin{array}{l}\text { un, l'uniforme } \\
\text { un, l'imprévu }\end{array}$ & l \\
\hline 4 & $\begin{array}{l}\text { politique } \\
\text { comique } \\
\text { étranger }\end{array}$ & $\begin{array}{l}\text { le politique } \\
\text { le comique } \\
\text { l'étranger }\end{array}$ & $\begin{array}{l}\text { le politique } \\
\text { le comique } \\
\text { l'étranger }\end{array}$ & $\begin{array}{l}\text { un, le politique } \\
\text { un, le comique } \\
\text { un, l'étranger }\end{array}$ & l \\
\hline 5 & blanc & le blanc & $\begin{array}{l}\text { le blanc } \\
(\ll \text { la semaine du blanc })\end{array}$ & $\begin{array}{l}\text { un, le blanc } \\
\text { (de poulet/dans } \\
\text { la conversation/ } \\
\text { être humain) }\end{array}$ & / \\
\hline 6 & $\begin{array}{l}\text { public } \\
\text { collectif }\end{array}$ & $\begin{array}{l}\text { le public } \\
\text { le collectif }\end{array}$ & le public (vs le privé) & $\begin{array}{l}1 \\
1\end{array}$ & $\begin{array}{l}\text { un, le public } \\
\text { un, le collectif }\end{array}$ \\
\hline
\end{tabular}

Tableau 1 
Avec cette catégorisation nous postulons une continuité pour certains items entre, d'un côté, des réalisations d'AS en discours et une lexicalisation nominale ( « série 3 » infra ${ }^{12}$ ) et, de l'autre, entre des réalisations discursives et une « série 2 », dans laquelle l'AS est stabilisé comme désignant un domaine ou une catégorie. En d'autres termes, les « séries » 2 et 3 correspondent à des étapes ultérieures potentielles des mécanismes représentés par l'AS, dont des exemples sont présentés ci-dessous sous « série 1 ».

\subsubsection{Exemples et discussion}

Voici quelques exemples. Nous les commentons en $\S 2.3$.

En série 1, des exemples d'AS : l'extraordinaire ; le merveilleux ; l'inoü̈; le flou; le vague (rester, regarder dans le vague); le chic (18); l'existant (24); l'historique (26) ; l'utile, le futile (29); le symbolique ; l'étranger; le tragique (le tragique de la situation); le baroque; le classique; l'intime, l'apparent, le substantiel, l'extérieur (28).

(28) Cette sorte de renversement des valeurs qui dans tous les ordres fera primer le réel sur le mot, l'intime et le substantiel sur l'extérieur et l'apparent (Maritain, Human. intégr., 1936).

(29) [le capitalisme] Il dépense 500 milliards de dollars par an pour injecter dans les « cerveaux humains disponibles » des substances publicitaires hallucinogènes conduisant à des confusions mentales entre le futile et l'utile, [...] (Politis, 11/07/2013, chronique)

Par définition, tous les Adj ou presque peuvent entrer dans la série 1 (cf. $\S$ 2.1.), mais certains n'en sortent pas, en particulier les Adj qualitatifs déjà présentés supra comme exprimant une subjectivité, un point de vue, parfois de manière hyperbolique (splendide, inouï, extraordinaire, ainsi que des Adj en in$\mathrm{X}$-able : irrémédiable) ; ils donnent parfois lieu à des emplois phraséologiques réguliers - des clichés (voir Lecolle 2012).

La série 2 rassemble des lexèmes nominaux qui conservent la forme d'AS, et dénomment un secteur, un domaine de référence, d'activité, comme on le voit d'après le cotexte dans les exemples (30), (31) et (32) ${ }^{13}$. Selon nous, ils relèvent de la catégorie nominale : en devenant des dénominations, ils s'affranchissent de l'Adj, même s'ils gardent un lien sémantique avec celui-ci : le judiciaire; le social ; l'économique ; le scolaire ; l'humanitaire ; l'étranger ; le religieux; le numérique (4); l'audiovisuel, le public, le privé (32); le baroque ; le classique ; le tragique; le romanesque (20); le merveilleux.

${ }^{12}$ Nous employons le mot série plutôt que catégorie, pour éviter la confusion avec d'autres sens de catégorie.

${ }^{13}$ Dans plusieurs des cas présentés ici, notre proposition rejoint l'analyse par ellipse (ellipse de «le domaine», « le secteur »), mais elle nous parait plus générale. 
(30) par incapacité [...] de me risquer dans un autre domaine que le scolaire. (Tournier)

(31) Qu'elles soient PDG ou dans l'humanitaire. (Angot)

(32) travailler dans le public ou dans le privé / dans l'audiovisuel (inventé)

La différence entre la série 1 et la série 2 peut être difficile à apprécier, mais voici quelques éléments :

- avec le tragique ${ }_{1}(\mathrm{AS})$ et le tragique 2 (Ndomaine) (idem merveilleux, romanesque), on observe que seul le tragique, peut entrer dans la structure [N1 de N2] proposée en 1.4 : le tragique de la situation n'a de sens qu'avec tragique $_{\text {Adj }}$ en AS ;

- observons l'exemple (20) : l'exagéré (AS avec Adj appréciatif) et le romanesque (dénomination de catégorie littéraire) y sont mis en parallèle, ce qui permet de constater la labilité de la frontière entre les deux séries, mais aussi de concevoir le passage de l'une à l'autre ;

- la comparaison des réalisations de littéraire en (33) et (34) permet également d'envisager ce passage potentiel - les exemples et l'analyse figurent dans Lecolle (2012).

(33) Oui, l’épithète littéraire ne doit pas être prise en mauvaise part, si le littéraire n'est que la mise en forme du commun. (Gurvitch, Traité de sociologie : t. 2, 1968)

(34) Si la pièce d'un écrivain moderne sur ce qu'a de mathématique la construction des colonnes du Parthénon relève du littéraire, c'est parce qu'il les appelle : « filles des nombres d'or « [...] (Benda, 1945)

De même, certains Adj régulièrement employés en AS comme des dénominations de catégories de pensée, de catégories descriptives en philosophie ou en sciences en particulier, nous paraissent à la limite d'une lexicalisation ${ }^{14}$ (le réel, l'infini (35)), et notamment lorsque ces AS fonctionnent en système d'opposition binaire : le matériel vs le spirituel (36); le singulier vs l'universel; le réel vs le virtuel.

(35) L'idée de l'infini est une des plus fondamentales de la nature humaine, si elle n'est pas toute la nature humaine; et pourtant l'homme ne fût point arrivé à comprendre dans sa réalité l'infini des choses, si l'étude expérimentale du monde ne l'y eût amené. (Renan, Avenir sc., 1890).

(36) La substitution du spirituel au matériel ne corrigerait pas l'erreur commise par la Renaissance (Carrel, 1935).

Enfin, signalons qu'une bonne partie des AS de la série 2 sont formés à partir d'Adj relationnels (judiciaire, politique, numérique, classique, matériel, spirituel...).

${ }^{14}$ Ils figurent comme Adj et Subst dans le TLFi, mais pas dans le dictionnaire de l'Académie. 
La série 3 représente le cas des lexèmes nominaux homonymes d'Adj l'acception nominale figure dans les dictionnaires d'usage mentionnés. La possibilité de l'article indéfini sert de test. Certains $\mathrm{N}$ peuvent désigner des humains $(+\mathrm{H})$, et être collectifs (+coll) : un historique ; un impondérable ; un manifeste ; un imprévu ; un bleu $\left(+\mathrm{H}^{15}\right)$; un blanc $(+\mathrm{H})$; un uniforme ; un fluide ; un liquide; un vague (à l'âme) ${ }^{16}$; un nécessaire (de toilette) (+coll) ; un collectif $(+$ coll $)$; un matériel $(+\mathrm{coll}) ;$ un religieux $(+\mathrm{H})$; un scolaire $(+\mathrm{H})$; un merveilleux $(+\mathrm{H})$; un humanitaire $(+\mathrm{H})$; un politique $(+\mathrm{H})$; un étranger $(+\mathrm{H})$; un comique $(+\mathrm{H})$; un public $(+\mathrm{H},+$ coll $) ; *$ un économique ; *un réel ; *un numérique ; *un audiovisuel; *un existant.

Les trois catégories décrites montrent la portée du «moule » constructionnel mentionné. Un même moule produit des résultats différents : fait discursif conjoncturel et/ou construction de classe notionnelle et/ou construction de lexème individuel ou collectif.

Le cas présenté en 4 nous ramène à des problématiques de morphologie constructionnelle, mais aussi de sémantique lexicale : nous comparons ici, d'un point de vue sémantique, des réalisations d'AS et de NQ dérivés avec ou sans affixe, donc relevant d'un autre procédé de construction (voir en § 1.1.1, les cas du type de vide $_{\text {subst, }}$ calme $e_{\text {subst }}$ ). Nous avons en effet rencontré des Adj qui, nominalisés, peuvent avoir (au moins) deux acceptions parallèles, l'une descriptible selon le sens que nous prêtons aux AS - '(catégorie de) ce qui est Adj' -, et l'autre selon celui d'un NQ - 'caractère d'Adj' ; 'le fait d'être Adj'. Ainsi chic $_{2}$, représenté en (37), a-t-il une signification différente de celle de chic $_{1}$ AS, vu en (18) - le chic $=$ 'ce qui est chic' :

(37) $[\ldots]$ tout mon goût va aux Françaises. Elles seules ont ce chic, et ce chien. (Aragon, 1936).

Mieux, ces significations sont prévisibles selon les deux « modèles », bien distincts, de l'AS et du NQ : à partir de chic ' 'caractère chic', on ne peut pas former la structure repérée en $\S 1.4$ pour chic $_{1}$ - ce qui est chic, c'est del c'est que. Mais, en revanche, le chic des Françaises; et on peut associer chic avec différents déterminants : démonstratif, comme en (37), ou indéfini (un chic).

On peut aussi confronter les deux modèles et les significations afférentes dans des cas où existe un NQ affixé (réel ${ }_{A S} /$ réalité $_{N Q}$, vrai ${ }_{A S} /$ vérité $_{N Q}$, fini $i_{A S}$ finitude $_{N Q}$ ). Mais la répartition n'est pas toujours celle attendue. Ainsi, pour

${ }^{15}$ Bleu ('un nouveau') et blanc ('un homme blanc') ont aussi, naturellement, d'autres acceptions. Nous revenons en 2.3 sur ces deux derniers N.

${ }^{16}$ Le TLF présente trois acceptions de vague substantivé, dans l'entrée « Adj et Subst ». Vague à l'âme est le seul qui relève de la série 3 (dans nos termes), les sens des deux autres (présentés ici en série 1) étant plus difficiles à saisir et même à distinguer. 
(le) réel $l_{A S}$, confronté à réalité : à la différence de blanc $_{A S}$ /blancheur $_{N Q}$, leurs significations manifestent indistinction et chevauchements, chacun empiétant sur le territoire de l'autre : en (38) ci-dessous, le réel s'interprète comme un $\mathrm{AS}$ - '(tout) ce qui est réel'. Mais pas en (40), où il peut être remplacé par la réalité. Quant à réel et réalité en (39), chacun des deux nous parait difficile à délimiter :

(38) Le virtuel est partout, mais la demande de réel augmente tout autant (Télérama, 11/2013)

(39) Qu'est-ce que le réel et de quelle façon perçoit-on la réalité ? (livre Le réel)

(40) Comment la science représente-elle le réel ? (livre Le réel)

À la différence de trouble, vide cités supra (§ 1.1.1) comme exemples de NQ convers, le réel ne satisfait pourtant pas aux tests de conversion :

? Cette créature est réelle / le réel de cette créature.

Malgré ce fait, il a bien, selon nous, une signification de NQ - 'caractère réel' -, tandis que réalité, de son côté, prend aussi les deux acceptions ('ce qui est réel' et 'caractère établi et fondé [...]' (TLFi)).

En définitive, alors que l'AS et le NQ ont structurellement des significations bien distinctes (le blanc vs la blancheur), on constate ici une convergence. Ce phénomène, lié au sens lexical des items, n'est certes pas général. Mais il nous parait être favorisé dans les cas de notions, de catégories de pensée (voir aussi le vrai/ la vérité), qui, loin d'être données, s'élaborent au contraire, par le langage ${ }^{17}$.

\subsection{CONTINUITÉ, PASSAGES, CHEVAUCHEMENTS}

Nous nous attardons à présent sur les items qui sont présents dans deux, voire trois des séries 1 à 3 (voir le tableau 1). Rappelons que nous nous fondons sur l'existant (telle réalisation) pour postuler le passage possible de 1 à 2 (e.g. numérique, tragique) et de 1 à 3 (e.g. imprévu). Certains items sont présents en 2 et 3 (e.g. politique) : nous considérons qu'ils procèdent tous deux de 1 , selon des processus différents.

Pour décrire les réalisations d'AS, leur devenir, et leur éventuelle répartition dans les séries 1 à 3 , nous distinguons deux niveaux théoriques de description : (i) un niveau syntaxique, celui de la « distorsion catégorielle », qui représente le potentiel du procédé, la «matrice »; (ii) un deuxième niveau lié au lexème adjectival lui-même. C'est, selon nous, à ce niveau et dans l'interaction entre le niveau (i) de la structure [le Adj] et celui (ii) de l'individu lexical Adj que se

${ }^{17}$ Ces considérations ne sont que des pistes : au-delà, il conviendrait d'étudier pour elles-mêmes ces répartitions, selon la signification des Adj et la présence ou l'absence dans le stock lexical de NQ, affixé ou non. 
pose la question des réalisations effectives et de la lexicalisation.

Le passage de 1 à 2 correspond à la stabilisation d'une notion dans un domaine structuré (celui du numérique, du public), ou un secteur d'analyse ou de pensée : de 'quelque chose de classique/ tragique/ baroque/ romanesque/ merveilleux' (qui a les caractéristiques portées par la signification de ces Adj - série 1) à 'ce qu'on peut ranger dans la catégorie (répertoriée) du classique/ tragique/ baroque/ romanesque/ merveilleux' - série 2. Concernant tragique, merveilleux, romanesque, cette mise en catégorie et en paradigme s'accompagne d'un « affadissement » du sens et même le provoque, en toute hypothèse, par l'objectivation de ce qui, fondamentalement, relevait de l'appréciatif. Quant à la série 3, nous postulons qu'elle procède de l'AS, par le biais d'une "singularisation », une discrétisation, ce que nous avons nommé un «prélèvement» sur le massif que représente la qualité : un blanc, un humanitaire, un nécessaire, un imprévu c'est 'un quelque chose qui est blanc/ humanitaire/ nécessaire/ imprévu' - un "quelque chose » qui peut aussi être un « quelqu'un» (cas de $+\mathrm{H}$ ), ou qui peut être une entité collective (public, matériel).

On peut naturellement se poser la question de la prévisibilité des réalisations en 2 et en 3. Nous avons esquissé ci-dessus quelques pistes en parlant (série 2) de domaines d'un côté, et de catégories de pensées (notions) de l'autre. Le premier cas (domaine) est sans doute balisé par la signification des Adj (nous avons mentionné les Adj relationnels catégorisants), mais aussi étroitement lié au monde, à la société et aux catégories qui y sont retenues (cf. Guilbert 1975) - domaine du numérique, de l'audiovisuel, catégories descriptives du tragique, du baroque, du romanesque, du merveilleux ; le second (notions) est peut-être plus directement prévisible, à partir du sens des Adj (infini, spirituel). Réciproquement, on peut aussi faire des suppositions sur ce qui ne sera pas retenu comme désignation de domaine ou de secteur (série 2) : l'outré, l'extraordinaire, l'inouï ont peu de chance de dénommer des catégories stables, et donc d'être des noms de domaines ou de secteur. Pourtant, le merveilleux et le tragique figurent dans cette série. Et peut-on parier sur l'exclusion, par exemple, de le gratuit, le substantiel?

La réflexion est la même, nous semble-t-il, pour le passage de 1 à 3 , où la difficulté est encore décuplée si l'on s'intéresse, non seulement à la prévisibilité de la réalisation de l'item nominal (un imprévu, un nécessaire, un politique, *un judiciaire), mais aussi à celle de sa signification (un merveilleux $(+\mathrm{H})$, un uniforme), même si certains sont prévisibles (un collectif (+coll)). De fait, la série 3 rassemble une grande diversité d'items nominaux, issus, par le biais de la «matrice» que constitue l'AS, de la rencontre entre la signification d'un Adj et une « réalité » à dénommer, dans le cadre de la société, de l'histoire 
et de la culture ; issus, donc, du croisement de phénomènes linguistiques et extralinguistiques.

Les propositions développées ici à propos de la construction de $\mathrm{N}$ désadjectivaux (série 3) se distinguent d'autres descriptions. Nous avons formulé précédemment nos réserves sur les explications en termes d'ellipse (voir l'exécutif vs le législatif en $§ 1.1 .2$ ). Signalons toutefois que ces réserves portent, non pas sur l'explication elle-même, mais sur sa généralisation. Nos propositions peuvent également être confrontées, à propos de $\mathrm{N}$ convers tels que bleu $_{\text {subst, }}$ blanc $_{\text {subst }}$, ou encore portable $e_{\text {subst }}$ à l'interprétation métonymique ou synecdochique en vigueur (cf. Corbin et Temple 1994, inter alii) : le N est construit à partir d'une propriété saillante de l'objet exprimée par l'Adj - Corbin D. \& Corbin P. parlent de « conversion de focalisation $»^{18}-$, propriété pour un ordinateur/un téléphone d'être portable, pour un fromage, un vêtement, etc. d'être bleu, pour un morceau de poulet ou une personne d'être blanc(he). Notre description est en fait logiquement réciproque de celle-ci : pour l'une, on part du référent pour expliquer son nom à partir d'une propriété saillante ; l'autre (la nôtre) postule, dans la nomination de tel objet, une discrétisation (singularisation) à partir d'une propriété continue. Ce raisonnement suppose la construction, fûtelle idéelle, d'une telle propriété, ce qui n'est pas pertinent pour tous les Adj (que serait la propriété « continue » d'être portable ?). Notre proposition ne couvre donc certainement pas la diversité des cas de nominalisations désadjectivales (voir des compléments chez Roché 1991, Schwarze 2012 et nos propositions dans Lecolle 2011 pour les Nhumains). Mais, avec celle de Lauwers (2008), elle est la seule, à notre connaissance, à établir une continuité et un lien sémantique entre plusieurs types de nominalisations avérées pour un même Adj, discursives et/ou lexicales.

Pour finir, on peut s'interroger sur les $\mathrm{N}$ dont le signifiant est présent dans les séries 2 et 3 (matériel, public, merveilleux, humanitaire, scolaire, étranger). Ces $\mathrm{N}$ sont de types différents : continu, massif et lié à l'Adj, d'un côté (en 2 -l'humanitaire), discret de l'autre (en 3 - un humanitaire). Mais leurs significations respectives ne sont pas sans relation : relation forte et facilement motivable entre l'humanitaire et un humanitaire (idem pour scolaire), relation forte aussi entre l'étranger et un étranger, relation plus lâche voire inexistante entre le merveilleux et un merveilleux - voir aussi le public (secteur) et un, le public (empirique). Il nous semble pourtant, dans tous les cas, que parler d'homonymie, comme le fait la morphologie lexématique lorsque des formes appartiennent à deux catégories, serait une simplification abusive, et que la

18 Voir Corbin D. \& Corbin P (1991), « Un traitement unifié du suffixe -ier(e)». Lexique, 10, cité par Koehl (2012). 
question de leur parenté sémantique, en lien avec l'Adj dont ils procèdent, doit, en tout état de cause, être posée.

Ces raisonnements plaident en définitive pour une approche des relations entre morphologie et sémantique en termes, non pas d'interface, ce qui suppose des domaines auto-suffisants et étanches, mais en termes de complémentarité et d'articulation des points de vue. Au-delà, la construction des objets (lexicaux, discursifs) que nous avons présentés est, selon nous, fondamentalement multifactorielle : y interviennent des faits d'ordre morphologique et syntaxique et d'ordre discursif, stylistique, rhétorique. Mais aussi des faits touchant le lexique en tant que tel, au sens où chaque unité lexicale est tributaire, d'une part de ce que permet et de ce à quoi oblige le système, et de ses relations avec d'autres unités lexicales, et, d'autre part, possède son cheminement et son histoire, en lien avec le référent et le contexte extra-linguistique. 


\section{BIBLIOGRAPHIE}

Apothéloz, D. (2003), « Le rôle de l'iconicité constructionnelle dans le fonctionnement du préfixe négatif in- », Cahiers de Linguistique Analogique, 1 : 35-63.

Bécherel D. (1979), « La substantivation des noms abstraits », Revue des langues Romanes, $83: 73-83$.

Colombat, B. (1992), «L'adjectif : perspectives historique et typologique. Présentation », Histoire Épistémologie Langage, 14/1 : 5-23.

Colombat, B. (1992), «L'adjectif dans la tradition latine : vers l'autonomisation d'une classe », Histoire Épistémologie Langage, 14/1 : 101-122.

Corbin, D. \& Temple, M. (1994), «Le monde des mots et des sens construits : catégories sémantiques, catégories référentielles », Cahiers de Lexicologie, 65 : 5-28.

Goes, J. (2011). «Les adjectifs primaires : entre quantité et qualité ». Studii de linguistică, $1: 121-137$.

Guilbert, L. (1975), La créativité lexicale, Paris : Larousse.

Kerleroux, F. (1996), La coupure invisible. Études de syntaxe et de morphologie, Paris : Presses universitaires du Septentrion.

Kerleroux, F. (1999), «Identification d'un procédé morphologique : la conversion », Faits de langues, Volume 7, numéro 14 : 89-100.

Koehl, A. (2012), La construction morphologique des noms désadjectivaux suffixés en français, thèse de doctorat en Sciences du Langage de l'université de Lorraine.

Lauwers, P. (2008), « The nominalization of adjectives in French: from morphological conversion to categorial mismatch », Folia linguistica, 42 : 135-176.

Lauwers, P. (à paraitre), « Between adjective and noun: category / function mismatch, constructional overrides and coercion », in Masini, F. \& Simone, R., Thematic issue from International Conference on «Word classes», Rome, March 2010, Amsterdam : Benjamins.

Lecolle, M. (2011), « Désadjectivaux formés par conversion et double catégorisation : le cas des adjectifs/noms en -aire », Revue Romane, 46-2 : 295-316.

Lecolle, M. (2012), « Néologie sémantique et néologie catégorielle : quelques propositions », Cahiers de Lexicologie, 100 : 81-104.

Lecolle, M. (à paraître), « Nominalisations désadjectivales en [le Adjectif]. Approche lexicale et sémantique », Le Français moderne, 2015.

Legallois, D. (2008), « Sur quelques caractéristiques des noms sous-spécifiés », Scolia, 23, 109-127.

Noailly, M. (1999), L’adjectif en français, Paris-Gap : Ophrys.

Noailly, M. (2004), « Du lien primordial de l'adjectif et du substantif en français, et du peu d'intérêt de la mise en relation de l'adjectif avec le verbe dans cette même 
langue », in François, J. \& Le Querler, N. (éds.) L'adjectif en français et à travers les langues, Caen : Presses Universitaires de Caen : 151-168.

Goes, J. (1993), « À la recherche d'une définition de l'adjectif », L'Information grammaticale, 58 : 11-14.

Riegel M. (2004), « La catégorie grammaticale de l'adjectif en français : entre référence et prédication ». In François, J. \& Le Querler, N. (éds.) L'adjectif en français et à travers les langues, Caen : Presses Universitaires de Caen : 181-205.

Riegel, M. (2013), « La sémantique des noms et les adjectifs substantivés « neutres » : l'essentiel et l'accessoire, faire du neuf avec du vieux, le plus drôle de l'histoire », communication Res per Nomen4, Résumé en ligne http://www.res-per-nomen.org/ respernomen/colloque-2013/Prgme_RPN4.pdf, consulté le 18/11/2013

Roché, M. (1991), De l'attribution du genre aux mots nouveaux dans la langue française, thèse, Toulouse.

Roché, M. (2005), « Sur une classe d'adjectifs par conversion », in I. Choi-Jonin, M. Bras, A. Dagnac et M. Rouquier (éd.), Questions de classification en linguistique : méthodes et descriptions, Berne : Peter Lang : 321-347.

Sablayrolles, J.-F. (2000), La Néologie en français contemporain : examen du concept et analyse de productions néologiques récentes, Paris : Champion.

Sanchez, J. et Cadiot, P. (2007), « Le cas des substantifs construits $\mathrm{X}_{\mathrm{A}}$-> suf.eur $\mathrm{Xeur}_{\mathrm{N}} /$ $\mathrm{X}_{\mathrm{A}} \rightarrow$ conv. $\mathrm{X}_{\mathrm{N}}$ : un point de vue sur la synonymie », Le Français moderne, 1 : 114-140.

Schwarze, C. (2012), « Le vert, les jeunes, l'important : aspects sémantiques de la conversion adjectif-nom », Scolia, $26: 153-170$.

Villalba, X. (2009), « Definite Adjective Nominalizations in Spanish ». M.T. Espinal, M. Leonetti \& L. Mc Nally (eds), Proceedings of the IV Nereus International Workshop "Definiteness and DP Structure in Romance Languages". Arbeitspapier 124. Fachbereich Sprachwissenschaft, Universität Konstanz : 139-153. 